\title{
Perceptual constancy in auditory perception of distance to railway tracks ${ }^{\text {a) }}$
}

\author{
Bert De Coensel ${ }^{\text {b) }}$ \\ Acoustics Group, Department of Information Technology, Ghent University, Ghent, Belgium \\ Mats E. Nilsson and Birgitta Berglund \\ Gösta Ekman Laboratory for Sensory Research, Karolinska Institutet and Stockholm University, Stockholm, \\ Sweden
}

\author{
A. L. Brown \\ Urban Research Program, Griffith School of Environment, Griffith University, Brisbane, Australia
}

(Received 2 October 2012; revised 31 March 2013; accepted 8 May 2013)

\begin{abstract}
Distance to a sound source can be accurately estimated solely from auditory information. With a sound source such as a train that is passing by at a relatively large distance, the most important auditory information for the listener for estimating its distance consists of the intensity of the sound, spectral changes in the sound caused by air absorption, and the motion-induced rate of change of intensity. However, these cues are relative because prior information/experience of the sound source-its source power, its spectrum and the typical speed at which it moves-is required for such distance estimates. This paper describes two listening experiments that allow investigation of further prior contextual information taken into account by listeners-viz., whether they are indoors or outdoors. Asked to estimate the distance to the track of a railway, it is shown that listeners assessing sounds heard inside the dwelling based their distance estimates on the expected train passby sound level outdoors rather than on the passby sound level actually experienced indoors. This form of perceptual constancy may have consequences for the assessment of annoyance caused by railway noise. (C) 2013 Acoustical Society of America. [http://dx.doi.org/10.1121/1.4807822]
\end{abstract}

PACS number(s): 43.66.Lj, 43.50.Qp, 43.50.Lj, 43.66.Qp [KVH]

Pages: 474-480

\section{INTRODUCTION}

The ability to accurately estimate the distance to a sound source solely based on auditory information is an important component of human spatial hearing. ${ }^{1}$ At short range, perception of distance provides the listener with information for spatial navigation or for preparing evasive action, ${ }^{2}$ which may be important for survival in darkened environments or when objects are outside the field of view. As a consequence of the importance of this ability for self-preservation, a large part of past research into the perception and the neuronal representation ${ }^{3}$ of auditory distance has focused on sound sources at relatively close distances from the head, up to a few meters. However, the distance to a sound source also plays an important role in effects on a larger spatial scale. For example, within several studies of community noise annoyance caused by railway noise, the distance to the railway track has been found to be a moderating factor. ${ }^{4,5}$ This may be explained, in part, by the rate of change in sound level caused by an approaching train, which is greater when the listener is located closer to the track. From an evolutionary perspective, sound sources that move toward the listener have a large biological saliency ${ }^{6}$ and may evoke intense emotional responses. ${ }^{7}$ The goal of this paper is to extend our

\footnotetext{
a) Portions of this work were presented in "Dwelling insulation as prior information in auditory distance perception of moving trains," in Proceedings of Euronoise 2012, Prague, Czech Republic, June 2012.

${ }^{b)}$ Author to whom correspondence should be addressed. Electronic mail: bert.decoensel@intec.ugent.be
}

knowledge of the acoustical cues that play a role in the perceived auditory distance to outdoor sound sources that are moving at a relatively long range, as well as on the influence of prior information.

It is well known that the auditory system uses several elements of acoustical information to estimate sound source distance. ${ }^{8}$ For stationary listeners and sound sources, the most important variables are the intensity of the sound, ${ }^{9}$ spectral changes due to air absorption, ${ }^{10}$ and, at close distance, interaural differences. ${ }^{11}$ Within enclosed environments with sound reflecting surfaces, important additional information is provided by the energy ratio of direct and reverberant sound ${ }^{12}$ and spectral changes due to the acoustical properties of reflecting surfaces. ${ }^{13}$ When the sound source is moving, the motion-induced rate of change of intensity or rise speed may also provide the listener with distance information. ${ }^{14}$ In addition, when the listener is moving, the change in direction of the sound source with respect to the change in direction of other stationary sources, or motion parallax, can be used to estimate sound source distance. $^{15}$

Based on the preceding, it can be expected that the most important auditory information for estimating the distance to outdoor sound sources that are moving at long range is formed by the intensity, the spectrum, and the rate of change of intensity of the sound. It is important to note that these auditory cues all depend on prior knowledge. In particular, prior information about the sound source such as its source power, its spectrum, or the typical speed at which it moves, and about the context in which the listening occurs, are 
required to estimate distance to the source. ${ }^{16,17}$ The present study considered the particular case of a listener situated in an at-home context, who is asked to estimate the distance to the track of a railway, based solely on auditory information of the passing trains. In contrast to earlier work in which virtual acoustics techniques have been applied to place sound sources at various distances, ${ }^{18,19}$ in this work, actual train passage recordings made in open field were used as experimental sounds. A first experiment (Sec. II A) presented the baseline condition: Participants were asked to estimate the distance to the railway track for train passages, binaurally recorded outdoors at varying distances from a railway, and presented through headphones. In a second experiment (Sec. II B), the sound field of trains passing by at varying distance was reproduced through loudspeakers placed outside a house while participants were seated inside the living room of the house. Results of both experiments are given in Sec. III. Conclusions are drawn from these results on the particular acoustical cues that are used to estimate the distance to the track as well as on the effect of prior contextual information about the dwelling.

\section{METHODOLOGY}

\section{A. Experiment 1}

\section{Experimental sounds}

The experimental sounds of the first experiment consisted of binaural recordings of train passages, made using a Brüel and Kjær type $4128 \mathrm{C}$ head and torso simulator (HATS) near a series of conventional railway tracks in The Netherlands and Austria at varying distance from the track and with trains driving at a wide range of speeds (but no high-speed trains). Recording locations were carefully selected on the basis of aerial photographs and visual inspection on the spot; only free field locations were selected where there were no buildings or noise barriers in the vicinity and where there was either grassland or plowed agricultural field in between the microphone and the railway track. From the many train pass-by recordings available, the 25 passages of highest quality were selected (based on the absence of sound from other sources, thus eliminating non-train sources that might have provided a reference for the absolute level). Subsequently, sounds spanning the complete pass-by were created, with a duration ranging from 11 to $75 \mathrm{~s}$. An overview of the main acoustical parameters of the selected experimental sounds can be found in Table I.

Figures 1 and 2 (diamond markers) show the actual distance to the railway track as a function of two important known cues for auditory distance perception: Sound intensity, as measured by the maximum A-weighted sound pressure level $L_{\mathrm{Amax}}$ of the sounds (fast time weighting, with a time constant of $125 \mathrm{~ms}$, is used throughout this paper), expressed in $\mathrm{dB}(\mathrm{A})$, and rate of change, as measured by the rise speed $v_{r}$ (see following text) of the sound level, expressed in $\mathrm{dB}(\mathrm{A}) / \mathrm{s}$. Note that the label $L_{\text {Amax }}^{\text {out }}$ is used to stress that the level is measured in outdoor environment. From both figures, it can be concluded that for experiment $1, v_{r}$ contains less information about distance (Pearson's $r=-0.56, p<0.01$, reducing
TABLE I. Maximum sound pressure level $\left(L_{\mathrm{Amax}}\right)$ and rise speed $\left(v_{r}\right)$ of the experimental sounds of experiment 1 (energetic averages between left and right ear), as measured outdoor (denoted with out), together with the actual distance $\left(d_{a c t}\right)$ to the track, the perceived auditory distance $\left(d_{e s t}\right)$ to the track ( $a=$ arithetic average, $g=$ geometric average, $l=$ lower quartile, $m=$ median, and $u=$ upper quartile of estimates), and the skewness $\left(\gamma_{\text {est }}\right)$ of the distribution of distance estimates (adjusted Fisher-Pearson standardized moment coefficient).

\begin{tabular}{|c|c|c|c|c|c|c|c|c|c|}
\hline Sound & $\begin{array}{c}L_{\mathrm{Amax}}^{\text {out }} \\
{[\mathrm{dB}(\mathrm{A})]}\end{array}$ & $\begin{array}{c}v_{r} \\
{[\mathrm{~dB}(\mathrm{~A}) / \mathrm{s}]}\end{array}$ & $\begin{array}{l}d_{a c t} \\
(\mathrm{~m})\end{array}$ & $\begin{array}{l}d_{e s t}^{a} \\
(\mathrm{~m})\end{array}$ & $\begin{array}{l}d_{e s t}^{g} \\
(\mathrm{~m})\end{array}$ & $\begin{array}{l}d_{e s t}^{l} \\
(\mathrm{~m})\end{array}$ & $\begin{array}{l}d_{e s t}^{m} \\
(\mathrm{~m})\end{array}$ & $\begin{array}{l}d_{e s t}^{u} \\
(\mathrm{~m})\end{array}$ & $\gamma_{\text {est }}$ \\
\hline 1 & 79.6 & 10.8 & 29.0 & 13.9 & 6.1 & 2.5 & 5.0 & 10.0 & 2.3 \\
\hline 2 & 78.0 & 10.3 & 29.0 & 29.5 & 20.5 & 10.0 & 20.0 & 37.5 & 1.7 \\
\hline 3 & 83.1 & 7.0 & 51.0 & 30.0 & 17.3 & 8.0 & 15.0 & 30.0 & 3.2 \\
\hline 4 & 64.9 & 2.5 & 63.0 & 136.7 & 75.8 & 40.0 & 55.0 & 200.0 & 1.4 \\
\hline 5 & 69.6 & 4.0 & 63.0 & 111.9 & 81.9 & 50.0 & 83.0 & 150.0 & 1.9 \\
\hline 6 & 69.2 & 8.2 & 57.0 & 144.6 & 84.6 & 35.0 & 80.0 & 200.0 & 3.6 \\
\hline 7 & 82.1 & 4.7 & 24.0 & 25.6 & 14.7 & 7.3 & 15.0 & 28.8 & 3.8 \\
\hline 8 & 84.1 & 5.6 & 24.0 & 18.9 & 11.4 & 5.0 & 10.0 & 25.0 & 2.5 \\
\hline 9 & 67.9 & 6.1 & 71.0 & 217.0 & 98.4 & 50.0 & 100.0 & 206.3 & 4.1 \\
\hline 10 & 72.0 & 4.6 & 108.0 & 365.5 & 140.4 & 52.5 & 150.0 & 272.5 & 4.3 \\
\hline 11 & 72.0 & 3.7 & 23.0 & 65.9 & 47.6 & 25.0 & 50.0 & 100.0 & 0.9 \\
\hline 12 & 93.6 & 19.0 & 14.0 & 5.9 & 3.1 & 1.0 & 3.0 & 5.0 & 3.9 \\
\hline 13 & 89.7 & 21.9 & 14.0 & 5.5 & 3.3 & 2.0 & 5.0 & 5.0 & 3.0 \\
\hline 14 & 53.8 & 5.8 & 336.0 & 494.0 & 365.2 & 237.5 & 375.0 & 750.0 & 0.7 \\
\hline 15 & 55.5 & 4.0 & 336.0 & 771.9 & 333.9 & 150.0 & 300.0 & 600.0 & 5.0 \\
\hline 16 & 59.7 & 5.4 & 152.0 & 399.4 & 225.8 & 105.0 & 250.0 & 737.5 & 0.8 \\
\hline 17 & 57.1 & 5.2 & 152.0 & 693.8 & 256.6 & 75.0 & 400.0 & 750.0 & 4.3 \\
\hline 18 & 59.8 & 5.2 & 152.0 & 226.9 & 157.4 & 80.0 & 175.0 & 300.0 & 0.8 \\
\hline 19 & 77.4 & 3.9 & 37.0 & 43.0 & 24.0 & 10.0 & 20.0 & 45.0 & 3.1 \\
\hline 20 & 76.3 & 6.6 & 150.0 & 369.5 & 126.5 & 80.0 & 100.0 & 200.0 & 4.8 \\
\hline 21 & 75.7 & 3.2 & 150.0 & 96.6 & 51.8 & 25.0 & 50.0 & 88.8 & 4.0 \\
\hline 22 & 83.6 & 9.8 & 25.0 & 39.2 & 21.0 & 10.0 & 20.0 & 45.0 & 2.2 \\
\hline 23 & 80.2 & 7.9 & 50.0 & 78.1 & 26.5 & 11.5 & 20.0 & 55.0 & 3.6 \\
\hline 24 & 77.7 & 9.4 & 100.0 & 151.9 & 60.3 & 27.5 & 60.0 & 87.5 & 2.6 \\
\hline 25 & 68.1 & 5.5 & 200.0 & 149.4 & 84.9 & 50.0 & 115.0 & 215.0 & 1.5 \\
\hline
\end{tabular}

to $r=-0.32, p>0.1$ if the two trains at closest distance are excluded from the analysis) than sound intensity (Pearson's $r=-0.82, p<0.001)$. This may be due to the rise speed being more affected by the combined effect of traveling speed of the train and the distance to the track than the sound intensity. It could therefore be expected that sound intensity would be the main variable for estimating distance in experiment 1 .

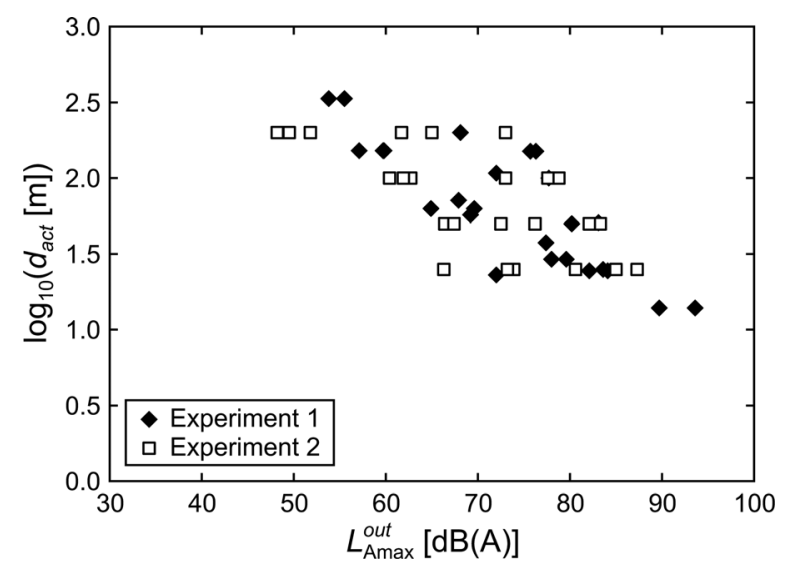

FIG. 1. Actual distance to the railway track $\left(d_{a c t}\right)$, as a function of $L_{\mathrm{Amax}}$ as measured outdoors, for all experimental sounds of both experiments. 


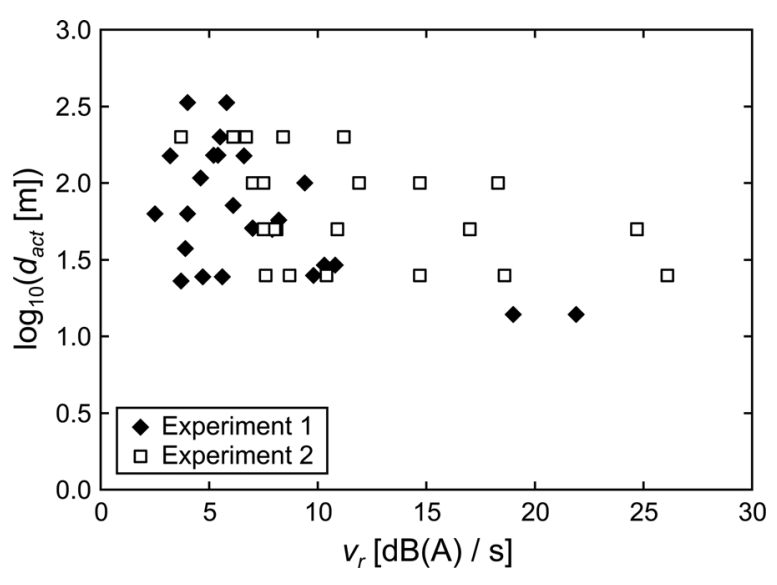

FIG. 2. Actual distance to the railway track $\left(d_{a c t}\right)$, as a function of rise speed $v_{r}$, for all experimental sounds of both experiments.

Furthermore, analysis pointed out a relatively low correlation (Pearson's $r=0.42, p<0.05$ ) between $L_{\text {Amax }}^{\text {out }}$ and rise speed in experiment 1 .

The procedure for calculating the rise speed of a train passage is illustrated in Fig. 3. First, the time series of the continuous equivalent sound pressure level $L_{\mathrm{Aeq}, 100 \mathrm{~ms}}$ of the passage is calculated with a time resolution of $100 \mathrm{~ms}$. Subsequently, a sliding window is used to find the largest increase in sound level during $1 \mathrm{~s}$. For the example of Fig. 3, this amounts to about $15 \mathrm{~dB}(\mathrm{~A}) / \mathrm{s}$. This procedure was found to be relatively robust, but nevertheless, calculated values were also checked visually.

\section{Participants and apparatus}

Seventy-five listeners (36 females, 39 males), with selfreported normal hearing, participated in the experiment. The sample size was chosen to be as large as practically feasible. The mean age of the listeners was $27.5 \mathrm{yr}$ (s.d. $=9.1$; range $=19-62) ; 80 \%$ of the listeners reported that they were not exposed to railway noise at home. The listeners were tested individually.

During the experiment, the participant was seated in a sound attenuating chamber. The experimental sounds were presented through open-type headphones (Sennheiser HD435), combined with a subwoofer placed in the experiment room to facilitate more accurate reproduction at low

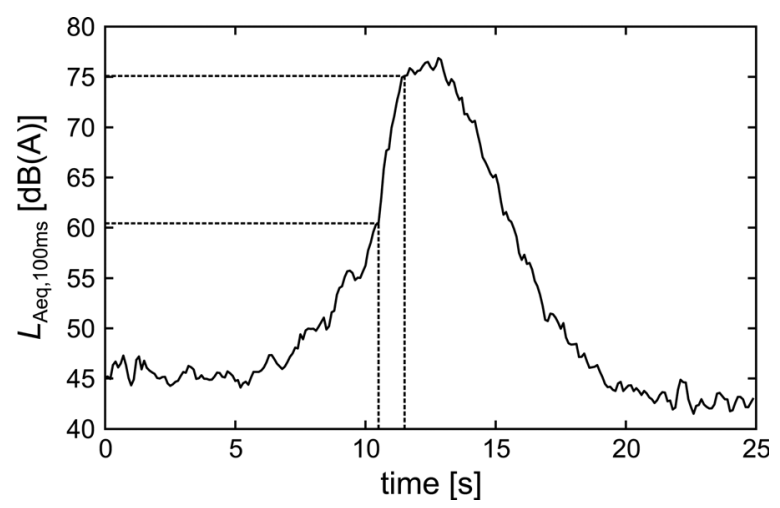

FIG. 3. Illustration of the procedure used to calculate the rise speed of a train passage. In this case, $v_{r} \approx 15 \mathrm{~dB}(\mathrm{~A}) / \mathrm{s}$. frequencies. The playback equipment was calibrated in 1/3-octave bands beforehand using the HATS system. The experimental sounds, that is, outdoor recordings of train passages, were presented at the sound levels measured in open field and reported in Table I. The presentation and response collection were controlled by a computer program (a set of MATLAB scripts with graphical user interface); no visual image of the setting was shown. All instruction and tests were conducted in Dutch.

\section{Procedure}

Before the start of the experiment, the participant was briefly informed about the experimental procedure, i.e., that he/she had to listen through headphones to the sound of train passages and that he/she had to guess the distance to the railway track. Although outdoor recordings were to be presented, the participant was not explicitly instructed to pretend to be sitting outdoors. During the experiment, the participant was asked to provide an absolute estimate of the distance for a selection of sounds by entering the distance (in $\mathrm{m}$ ) on a numeric keypad. The participant was free to listen to each presented sound as many times as needed. Due to time constraints and limits caused by fatigue (the present test was part of a larger experiment, which had a duration of about $1 \mathrm{~h}$ for each participant), it was not feasible to present each of the 25 experimental sounds to each participant. Therefore each participant was presented a sequence of eight randomly selected sounds. Consequently, for each experimental sound, the auditory distance was estimated on average by 24 different participants.

\section{B. Experiment 2}

The second experiment was part of a larger experiment on noise annoyance caused by different types of trains. A complete description of the methodology of this experiment and the selection of participants can be found in a previous paper. $^{20}$ Here we will briefly summarize the methodology with a focus on those parts that are most important for this work.

\section{Experimental sounds}

In experiment 2 , the experimental sounds consisted of two-channel recordings of train pass-by noise, performed near a series of railway tracks in Belgium, France, The Netherlands, and Germany. Recordings were made at varying distance from the track, and different types of trains were considered (conventional trains, high-speed trains, and trains based on magnetic levitation), driving at various speeds ranging up to $400 \mathrm{~km} / \mathrm{h}$. All recordings for experiment 2 were also performed in open space locations without noise barriers selected according to the same principles as for experiment 1 . The 24 pass-by recordings of highest quality were selected, and sounds spanning the complete pass-by were created, each with a duration of $45 \mathrm{~s}$. An overview of the main acoustical parameters of the selected experimental sounds can be found in Table II (outdoor values denoted with out). 
TABLE II. Maximum sound pressure level $\left(L_{\mathrm{Amax}}\right)$ and rise speed $\left(v_{r}\right)$ of the experimental sounds of experiment 2 (energetic averages between left and right ear), as measured outdoor (denoted with out) as well as indoor (denoted with in), together with the actual distance $\left(d_{a c t}\right)$ to the track, the perceived auditory distance $\left(d_{e s t}\right)$ to the track ( $a=$ arithetic average, $g=$ geometric average, $l=$ lower quartile, $m=$ median, and $u=$ upper quartile of estimates), and the skewness $\left(\gamma_{\text {est }}\right)$ of the distribution of distance estimates (adjusted Fisher-Pearson standardized moment coefficient).

\begin{tabular}{|c|c|c|c|c|c|c|c|c|c|c|}
\hline Sound & $\begin{array}{c}L_{\mathrm{Amax}}^{\text {out }} \\
{[\mathrm{dB}(\mathrm{A})]}\end{array}$ & $\begin{array}{c}L_{\mathrm{Amax}}^{i n} \\
{[\mathrm{~dB}(\mathrm{~A})]}\end{array}$ & $\begin{array}{c}v_{r} \\
{[\mathrm{~dB}(\mathrm{~A}) / \mathrm{s}]}\end{array}$ & $\begin{array}{l}d_{a c t} \\
(\mathrm{~m})\end{array}$ & $\begin{array}{l}d_{e s t}^{a} \\
(\mathrm{~m})\end{array}$ & $\begin{array}{l}d_{e s t}^{g} \\
(\mathrm{~m})\end{array}$ & $\begin{array}{l}d_{e s t}^{l} \\
(\mathrm{~m})\end{array}$ & $\begin{array}{l}d_{e s t}^{m} \\
(\mathrm{~m})\end{array}$ & $\begin{array}{l}d_{e s t}^{u} \\
(\mathrm{~m})\end{array}$ & $\gamma_{e s t}$ \\
\hline 1 & 66.3 & 53.3 & 7.6 & 25.0 & 307.3 & 152.3 & 67.5 & 160.0 & 300.0 & 6.5 \\
\hline 2 & 72.5 & 59.4 & 8.1 & 50.0 & 514.0 & 174.1 & 50.0 & 178.0 & 500.0 & 3.3 \\
\hline 3 & 60.4 & 47.5 & 7.3 & 100.0 & 645.6 & 287.3 & 118.8 & 250.0 & 700.0 & 8.0 \\
\hline 4 & 48.2 & 35.0 & 3.7 & 200.0 & 969.6 & 544.0 & 200.0 & 575.0 & 1000.0 & 2.0 \\
\hline 5 & 73.9 & 59.6 & 10.4 & 25.0 & 195.1 & 89.2 & 40.0 & 78.0 & 200.0 & 2.2 \\
\hline 6 & 80.6 & 68.0 & 14.7 & 25.0 & 138.1 & 55.7 & 20.0 & 50.0 & 162.5 & 2.7 \\
\hline 7 & 87.3 & 74.1 & 18.6 & 25.0 & 107.5 & 27.6 & 10.0 & 20.0 & 72.5 & 6.2 \\
\hline 8 & 66.4 & 51.6 & 8.0 & 50.0 & 269.1 & 143.6 & 67.5 & 100.0 & 325.0 & 3.5 \\
\hline 9 & 76.2 & 63.2 & 10.9 & 50.0 & 196.8 & 76.7 & 30.0 & 75.0 & 200.0 & 4.2 \\
\hline 10 & 83.3 & 69.8 & 17.0 & 50.0 & 78.5 & 30.5 & 10.0 & 25.0 & 77.5 & 3.7 \\
\hline 11 & 62.7 & 48.6 & 7.0 & 100.0 & 370.0 & 174.4 & 75.0 & 190.0 & 400.0 & 3.6 \\
\hline 12 & 73.0 & 60.7 & 14.7 & 100.0 & 232.9 & 102.7 & 50.0 & 100.0 & 200.0 & 3.6 \\
\hline 13 & 78.8 & 65.7 & 18.3 & 100.0 & 170.6 & 70.4 & 23.8 & 50.0 & 200.0 & 2.2 \\
\hline 14 & 49.5 & 35.0 & 6.4 & 200.0 & 768.4 & 392.5 & 152.5 & 325.0 & 1000.0 & 3.4 \\
\hline 15 & 61.7 & 49.3 & 6.7 & 200.0 & 503.7 & 247.2 & 100.0 & 200.0 & 550.0 & 3.0 \\
\hline 16 & 65.0 & 51.7 & 8.4 & 200.0 & 342.1 & 161.0 & 60.0 & 150.0 & 400.0 & 2.6 \\
\hline 17 & 73.2 & 60.6 & 8.7 & 25.0 & 163.5 & 84.3 & 47.5 & 100.0 & 200.0 & 3.3 \\
\hline 18 & 85.0 & 71.4 & 26.1 & 25.0 & 65.7 & 26.0 & 10.0 & 20.0 & 50.0 & 4.8 \\
\hline 19 & 67.4 & 54.3 & 7.5 & 50.0 & 314.1 & 165.3 & 73.8 & 150.0 & 400.0 & 3.5 \\
\hline 20 & 82.1 & 68.6 & 24.7 & 50.0 & 140.1 & 42.3 & 15.0 & 50.0 & 100.0 & 4.2 \\
\hline 21 & 61.9 & 49.1 & 7.5 & 100.0 & 413.2 & 231.4 & 100.0 & 200.0 & 500.0 & 2.6 \\
\hline 22 & 77.6 & 64.5 & 11.9 & 100.0 & 183.5 & 63.3 & 25.0 & 50.0 & 150.0 & 3.9 \\
\hline 23 & 51.8 & 38.9 & 6.1 & 200.0 & 875.6 & 456.0 & 200.0 & 500.0 & 1000.0 & 4.7 \\
\hline 24 & 73.0 & 59.8 & 11.2 & 200.0 & 301.2 & 123.9 & 50.0 & 120.0 & 300.0 & 3.9 \\
\hline
\end{tabular}

Figures 1 and 2 (rectangle markers) show the actual distance to the railway track, as a function of the $L_{\text {Amax }}$ (Pearson's $r=-0.66, \quad p<0.001$ ) and rise speed $v_{r}$ (Pearson's $r=-0.45, p<0.05)$ of the sounds. Note that the levels reported in previous work $^{20}$ are façade levels, which are approximately $3 \mathrm{~dB}(\mathrm{~A})$ higher than the outdoor free field levels given in Table II and shown in Fig. 1. It can be seen that the range in $L_{\text {Amax }}^{\text {out }}$ is similar to the range covered in experiment 1 , but the presence of high-speed trains obviously results in a larger number of trains with a high rise speed. Furthermore, analysis showed that $L_{\text {Amax }}^{\text {out }}$ and rise speed were highly correlated in experiment 2 (Pearson's $r=0.82, p<0.001$ ).

\section{Participants and apparatus}

A group of 100 participants (49 females, 51 males), with self-reported normal hearing, was recruited to be representative of the Dutch population on factors such as age, gender, educational level, general health, and noise sensitivity. The sample size was chosen to be as large as practically feasible. The mean age of the participants was $45.1 \mathrm{yr}$ (s.d. $=13.4$; range $=21-69) ; 69 \%$ of the listeners reported that they were not exposed to railway noise at home; this corresponds to the nationwide average of the Dutch population. ${ }^{20}$

During the experiment, participants were seated in the living room of a house situated in a quiet environment, approximating a real-life at-home situation. To create a realistic indoor sound field, produced by a moving train outside the house, the experimental sounds were played back through four loudspeakers (stacked two by two) and a large subwoofer placed outside the house (invisible to the panelists). A sound level meter was placed outdoors in front of the façade of the living room, and the playback equipment was carefully calibrated in 1/3-octave bands, spanning the frequency range from $30 \mathrm{~Hz}$ to $16 \mathrm{kHz}$, such that the sound levels measured at the façade corresponded to the measured sound levels in free field plus $3 \mathrm{~dB}$. During the experiments, one window of the living room was slightly opened, resulting in an insulation of the house of about $13 \mathrm{~dB}(\mathrm{~A})$, i.e., the difference between the level of the train passages outdoors (measured façade level minus $3 \mathrm{~dB}$ ) and indoors (measured using a HATS placed among the participants). Note that the living room was not square, not all walls were straight, and there was large furniture located against the walls; there were no indications that there would be a problem with room modes. A picture of the playback system is shown in Fig. 4. The resulting sound levels inside the living room are given in Table II (indoor values denoted with in). In the remainder of this work, we will use $L_{\text {Amax }}^{i n}$ to explicitly refer to the sound levels as measured inside the living room. As in experiment 1, the presentation of sounds was controlled through MATLAB.

\section{Procedure}

The listening experiment was performed in sessions during which four to six participants were seated in the living 


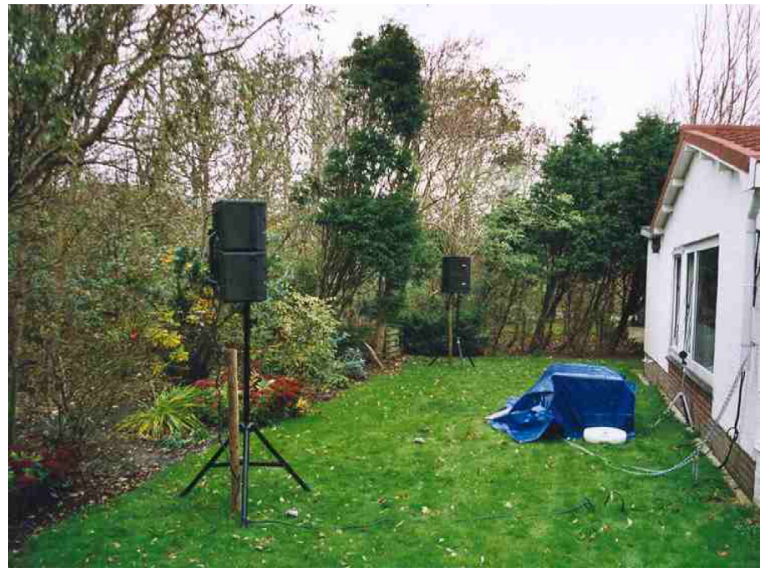

FIG. 4. (Color online) Playback system used in experiment 2.

room of the house. A single session had a duration of about $4 \mathrm{~h}$ and consisted of two parts. First, an experiment on annoyance caused by transportation noise was carried out in which participants performed daily activities such as reading a magazine or having a light conversation; results for this part are reported in earlier work. ${ }^{20}$ Second, a more conventional listening experiment was carried out in which all sounds were presented sequentially, in random order, and in which perceived auditory distance to the track was assessed. After each train passage, the participants were asked to write down their impression of the distance (in $\mathrm{m}$ ) from the house to the railway track on a sheet of paper.

\section{RESULTS}

\section{A. Accuracy in estimated distance}

For each of the sounds of both experiments, the arithmetic mean, geometric mean, and median of the estimated distances were calculated; values can be found in Tables I and II, together with the lower quartiles, upper quartiles and skewness of the distribution of distance estimates. It was found that for both experiments, the distributions of distance estimates were strongly positively skewed. On average, the arithmetic means were larger than the medians by a factor of 2.0 for experiment 1 and by a factor of 2.6 for experiment 2 . For both experiments, the geometric mean and median perceived distance to the track showed the best correspondence with the actual distance (no significant difference between both measures is found); the arithmetic mean generally overestimated the actual distance. In the following analysis, median values will be used, in accordance with previous studies; ${ }^{17}$ the label $m$ will be dropped from here on.

Figure 5 shows the (logarithm of the) median perceived distance as a function of the (logarithm of the) actual distance for all sounds of both experiments. A slightly better consistency in estimating distances is found for experiment 1; Pearson's correlation between median perceived and actual distance is $r=0.89$ for experiment 1 and $r=0.64$ for experiment 2 ( $p<0.001$ for both). Overall, the results show a more or less equal level of performance in estimating distances between both experiments. At the short end of the distance scale, the sounds presented in experiment 1 were

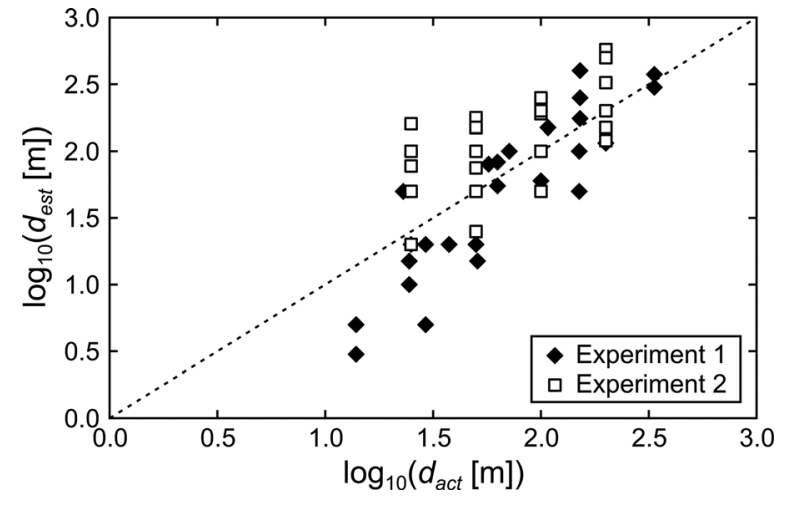

FIG. 5. Median perceived egocentric auditory distance $\left(d_{\text {est }}\right)$ as a function of actual distance $\left(d_{a c t}\right)$, for all sounds of both experiments.

mainly estimated to be too near. On the other hand, the sounds presented in experiment 2 were mainly estimated to be too far away. Experiment 2 had more trains with a high rise speed, thus, the existence of higher rise speeds did not make train passages sound nearer. Finally, it can be seen that there was negligible difference between experiments in terms of spread in perceived distance for a given actual distance, at least for all except the nearest actual distances.

\section{B. Influence of acoustical parameters}

Figure 6 shows, for all sounds of both experiments, the perceived auditory distance as a function of the maximum A-weighted sound pressure level as measured at the location of the ear of the listener $\left(L_{\text {Amax }}^{\text {ear }}\right.$, i.e., $L_{\text {Amax }}^{\text {out }}$ for experiment 1 and $L_{\text {Amax }}^{\text {in }}$ for experiment 2 ). The perceived auditory distance was found to be strongly negatively correlated to sound level; Pearson's correlation between median perceived distance and $L_{\text {Amax }}^{e a r}$ was $r=-0.92$ for experiment 1 and $r=-0.94$ for experiment 2 ( $p<0.001$ for both). These results suggest that perceived loudness, as measured by $L_{\text {Amax }}$, was the main variable used for distance assessment in both experiments.

For equal sound levels, as presented at the ear of the listener, the sounds of experiment 2 were perceived to be closer than those of experiment 1 by almost one order of

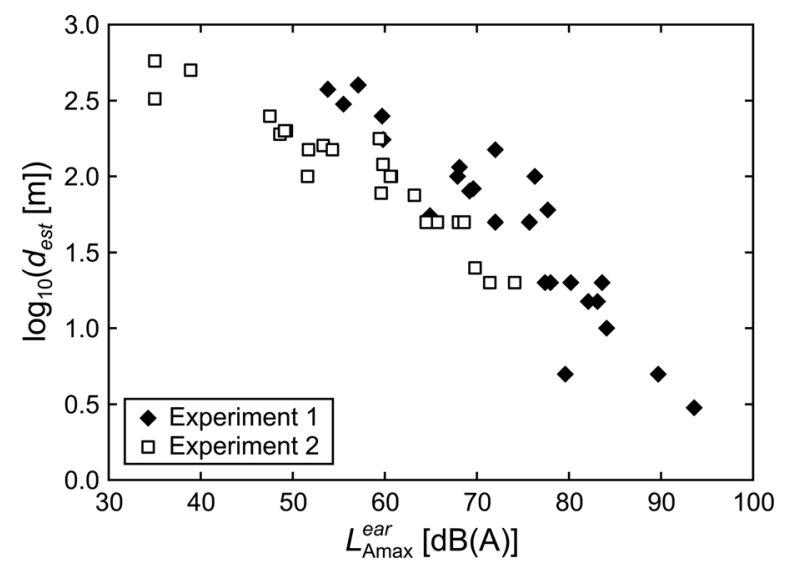

FIG. 6. Median perceived egocentric auditory distance $\left(d_{e s t}\right)$, as a function of $L_{\mathrm{Amax}}$ at the location of the ear of the listener, for all sounds of both experiments. 
magnitude. One could argue that this may be due to spectral differences between the sounds of both experiments. However, the indoor sounds of experiment 2 contained less energy at high frequencies because they had been filtered by the building envelope and, based on this argument, should have sounded further away at equal sound level. When results were plotted against outdoor levels, as shown in Fig. 7 , the difference between the results of both experiments disappeared. This suggests that the participants of experiment 2 were taking into account the fact that they were listening to sounds that had been filtered by the dwelling when estimating the distance based on sound level.

To quantify the difference shown in Fig. 6 between both experiments, a linear regression analysis was performed with $L_{\text {Amax }}^{e a r}$ as dependent variable, and the (logarithm of the) median perceived distance as independent variable, for the pooled data of both experiments. An independent-samples $t$ test (unequal sample sizes, unequal variance) was then conducted to compare the error terms of the estimated $L_{\text {Amax }}^{e a r}$ for both experiments. There was a significant difference between the error terms for the subset of data of experiment 1 (mean $=4.18$, s.d. $=5.46)$ and the subset of data of experiment 2 (mean $=-4.35$, s.d. $=3.94), t(44)=6.29$ $(p<0.001)$, indicating a horizontal shift of $8.5 \mathrm{~dB}(\mathrm{~A})$ in Fig. 6 between the data of both experiments.

Finally, Fig. 8 shows the perceived auditory distance as a function of the rise speed $v_{r}$ of the sounds. As expected, for experiment 1 , when the two train passages at close distance are not taken into account, there was no clear relationship between $v_{r}$ and perceived auditory distance. For experiment 2, it was assumed that the rise speed measured inside the dwelling equals the rise speed measured outside the dwelling. In this case, a good correlation between $d_{e s t}$ and $v_{r}$ was found (Pearson's $r=-0.85$, Spearman's $\rho=-0.94, p<0.001$ for both), although the correlation with $L_{\text {Amax }}^{\text {out }}$ was more linear and pronounced (Pearson's $r=-0.95$, Spearman's $\rho=-0.98, p<0.001$ for both). A sequential multiple linear regression analysis was performed with $d_{e s t}$ as dependent variable, and $L_{\text {Amax }}^{\text {out }}$ and $v_{r}$ as independent variables. It was found that a model containing only $L_{\text {Amax }}^{\text {out }}$ explained $90.0 \%$ of variance in median auditory distance estimates. When $v_{r}$ was added as independent variable,

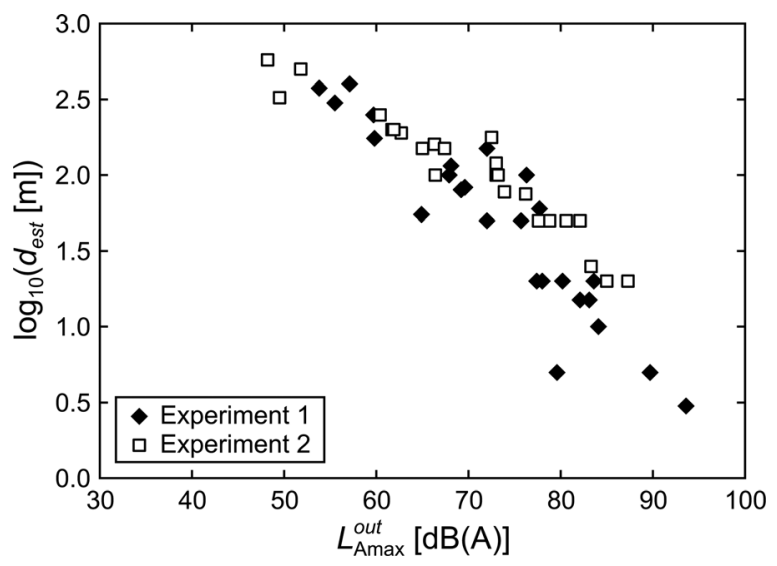

FIG. 7. Median perceived egocentric auditory distance $\left(d_{\text {est }}\right)$, as a function of $L_{\text {Amax }}$ as measured outdoors, for all sounds of both experiments.

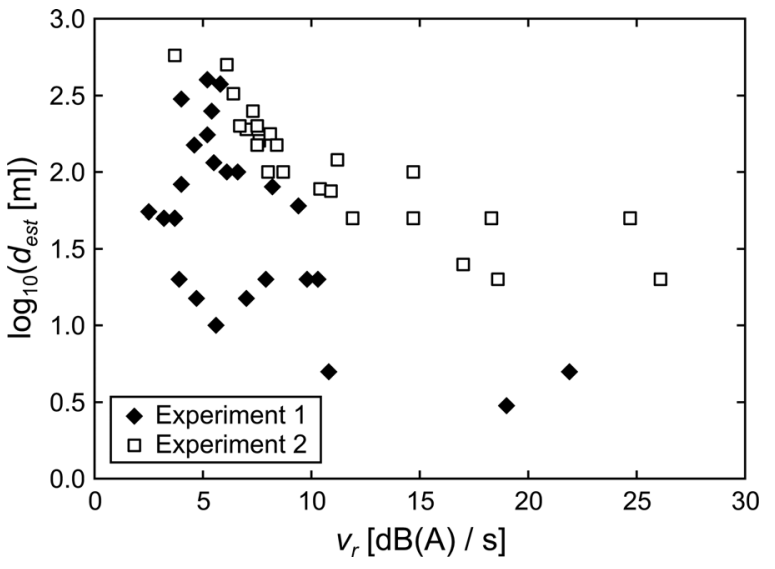

FIG. 8. Median perceived egocentric auditory distance $\left(d_{e s t}\right)$, as a function of rise speed $v_{r}$, for all sounds of both experiments.

the variance explained increased to $91.0 \%$, but the change in F-value was not significant $(p>0.1)$, so $v_{r}$ did not explain a significant additional amount of variance.

\section{DISCUSSION AND CONCLUSIONS}

In this paper, two experiments were presented in which the distance to the track of passing trains had to be estimated based only on auditory information. In the first experiment, train passages recorded outdoors were presented through headphones; in the second experiment, train passages were played back outside a house while the participants were seated inside the house. Although individual distance estimates showed high variability, median distance estimates were in good agreement with actual distances for both experiments. The effect of two important auditory cues that are known to influence perceived distance to outdoor sound sources that are moving at long range (maximum sound level and sound level rise speed) was investigated, and it was found, in both experiments, that the maximum sound level explained most of the variance in estimated distance. However, of particular interest was the finding that for equal maximum sound levels at the ear of the listeners, the trains in the second experiment were perceived to be closer than those in the first experiment, but when the outdoor levels of these train passages were examined, the differences disappeared.

We suggest that this result could be explained as a form of perceptual constancy, ${ }^{21,22}$ whereby individuals base their distance estimates on the sound level they would expect to experience outside the dwelling rather than on the sound level actually experienced indoors. The latter is, of course, lower as a result of the attenuation of the building envelope. Interestingly, similar perceptual constancy effects have been suggested in the case of noise annoyance for changes in the sound level over time ${ }^{23,24}$ as well as for judgments of the differences between indoor and outdoor sound levels. ${ }^{25}$

The authors acknowledge that differences in experimental sounds and reproduction techniques between both experiments could be considered to be confounding factors; therefore much care was taken in the implementation of both experiments to avoid this possibility. Recording locations 
and experimental sounds were selected based on the same principles for both experiments. Subsequently, care was taken to achieve accurate reproduction (at-the-ear for the first experiment and at the façade for the second experiment) by calibrating the playback equipment in 1/3-octave bands. Consequently, differences between the at-the-ear signals of both experiments are predominantly due to the attenuation of the building envelope and the effect of the room (i.e., due to being located indoors). For these reasons, it is argued that the observed at-the-ear level differences between sounds assessed equal in distance in both experiments is not attributable to differences in experimental sounds and reproduction techniques between the experiments.

It is generally acknowledged that having access to both a quiet indoor and outdoor section of the dwelling is essential for general well-being. ${ }^{26}$ The results of this paper can thus be interpreted as additional justification of the use of façade levels to assess the effects of environmental noise in indoor situations. ${ }^{25}$ However, the indoor level still determines the probability that sounds originating outdoors are audible and noticed when inside; this is essential for annoyance to emerge. ${ }^{27}$ A comprehensive assessment of noise annoyance in indoor situations should therefore also account for the noticeability of sounds; ${ }^{28}$ once a sound is noticed, it may be appraised on the basis of the expected outdoor level and the information present in the sound ${ }^{29}$ instead of the actual indoor level.

\section{ACKNOWLEDGMENTS}

B.D.C. is a postdoctoral fellow of the Research Foundation-Flanders (FWO-Vlaanderen); the support of this organization is gratefully acknowledged. The authors would also like to thank Peter Lercher for providing part of the sounds used in Experiment 1, and Gary J. Raw for providing information regarding the occurrence of perceptual constancy in noise annoyance.

${ }^{1}$ P. Zahorik, D. S. Brungart, and A. W. Bronkhorst, "Auditory distance perception in humans: A summary of past and present research," Acta Acust. Acust. 91, 409-420 (2005).

${ }^{2}$ L. D. Rosenblum, A. P. Wuestefeld, and H. M. Saldana, "Auditory looming perception: Influences on anticipatory judgments," Perception 22, 1467-1482 (1993).

${ }^{3}$ M. S. A. Graziano, L. A. J. Reiss, and C. G. Gross, “A neuronal representation of the location of nearby sounds," Nature 397, 428-430 (1999).

${ }^{4}$ T. Morihara, T. Sato, and T. Yano, "Comparison of dose-response relationships between railway and road traffic noises: The moderating effect of distance," J. Sound Vib. 277, 559-565 (2004).

${ }^{5}$ A. Preis and R. Gołebiewski, "Noise annoyance perception as a function of distance from a moving source," Noise Control Eng. J. 52, 20-25 (2004).

${ }^{6} \mathrm{~J}$. G. Neuhoff, "An adaptive bias in the perception of looming auditory motion," Ecol. Psychol. 13, 87-110 (2001).
${ }^{7}$ A. Tajadura-Jiménez, A. Väljamäe, E. Asutay, and D. Västfjäll, "Embodied auditory perception: The emotional impact of approaching and receding sound sources," Emotion 10, 216-229 (2010).

${ }^{8}$ D. H. Mershon and J. N. Bowers, "Absolute and relative cues for the auditory perception of egocentric distance," Perception 8, 311-322 (1979).

${ }^{9}$ P. D. Coleman, "An analysis of cues to auditory depth perception in free space,” Psychol. Bull. 60, 302-315 (1963).

${ }^{10}$ A. D. Little, D. H. Mershon, and P. H. Cox, "Spectral content as a cue to perceived auditory distance," Perception 21, 405-416 (1992).

${ }^{11}$ D. S. Brungart, N. I. Durlach, and W. M. Rabinowitz, "Auditory localization of nearby sources. II. Localization of a broadband source," J. Acoust. Soc. Am. 106, 1956-1968 (1999).

${ }^{12}$ Y.-C. Lu and M. Cooke, "Binaural estimation of sound source distance via the direct-to-reverberant energy ratio for static and moving sources," IEEE Trans. Audio Speech Lang. Process. 18, 1793-1805 (2010).

${ }^{13}$ R. A. Butler, E. T. Levy, and W. D. Neff, "Apparent distance of sounds recorded in echoic and anechoic chambers," J. Exp. Psychol. Hum. Percept. Perform. 6, 745-750 (1980).

${ }^{14}$ C. Pörschmann and C. Störig, "Investigations into the velocity and distance perception of moving sound sources," Acta Acust. Acust. 95, 696-706 (2009).

${ }^{15}$ D. H. Ashmead, D. L. Davis, and A. Northington, "Contribution of listeners' approaching motion to auditory distance perception,” J. Exp. Psychol. Hum. Percept. Perform. 21, 239-256 (1995).

${ }^{16} \mathrm{P}$. D. Coleman, "Failure to localize the source distance of an unfamiliar sound," J. Acoust. Soc. Am. 34, 345-346 (1962).

${ }^{17}$ J. W. Philbeck and D. H. Mershon, "Knowledge about typical source output influences perceived auditory distance," J. Acoust. Soc. Am. 111, 1980-1983 (2002).

${ }^{18} \mathrm{P}$. Zahorik, "Assessing auditory distance perception using virtual acoustics,” J. Acoust. Soc. Am. 111, 1832-1846 (2002).

${ }^{19}$ G. Kearney, M. Gorzel, H. Rice, and F. Boland, "Distance perception in interactive virtual acoustic environments using first and higher order ambisonic sound fields," Acta Acust. Acust. 98, 61-71 (2012).

${ }^{20}$ B. De Coensel, D. Botteldooren, B. Berglund, M. E. Nilsson, T. De Muer, and P. Lercher, "Experimental investigation of noise annoyance caused by high-speed trains," Acta Acust. Acust. 93, 589-601 (2007).

${ }^{21}$ P. Zahorik and F. L. Wightman, "Loudness constancy with varying sound source distance," Nat. Neurosci. 4, 78-83 (2001).

${ }^{22}$ Y.-K. Min and D. H. Mershon, "An adjacency effect in auditory distance perception," Acta Acust. Acust. 91, 480-489 (2005).

${ }^{23}$ G. J. Raw and I. D. Griffiths, "Subjective response to changes in road traffic noise: A model," J. Sound Vib. 141, 43-54 (1990).

${ }^{24}$ A. L. Brown and I. van Kamp, "Response to a change in transport noise exposure: Competing explanations of change effects," J. Acoust. Soc. Am. 125, 905-914 (2009).

${ }^{25}$ M. E. Nilsson and B. Berglund, "Noise annoyance and activity disturbance before and after the erection of a roadside noise barrier," J. Acoust. Soc. Am. 119, 2178-2188 (2006).

${ }^{26} \mathrm{E}$. Öhrström, "Longitudinal surveys on effects of changes in road traffic noise-annoyance, activity disturbances, and psycho-social well-being," J. Acoust. Soc. Am. 115, 719-729 (2004).

${ }^{27}$ B. De Coensel, D. Botteldooren, T. De Muer, B. Berglund, M. E. Nilsson, and P. Lercher, "A model for the perception of environmental sound based on notice events," J. Acoust. Soc. Am. 126, 656-665 (2009).

${ }^{28}$ A. J. Torija, D. P. Ruiz, B. De Coensel, D. Botteldooren, B. Berglund, and A. Ramos-Ridao, "Relationship between road and railway noise annoyance and overall indoor sound exposure," Transport. Res. D 16, 15-22 (2011).

${ }^{29} \mathrm{~K}$. Genuit, "The problem of predicting noise annoyance as a function of distance," in Proceedings of the 17th International Conference on Acoustics (ICA), Rome, Italy, 2001 (on CD rom). 